“C 2016 IEEE. Personal use of this material is permitted. Permission from IEEE must be obtained for all other uses, in any current or future media, including reprinting/republishing this material for advertising or promotional purposes, creating new collective works, for resale or redistribution to servers or lists, or reuse of any copyrighted component of this work in other works." 


\section{A Novel Approach of Mobility Management for the D2D Communications in 5G Mobile Cellular Network System}

\author{
Shouman Barua \\ University of Technology, Sydney \\ Australia \\ Email: Shouman.Barua@uts.edu.au
}

\author{
Professor Robin Braun \\ University of Technology, Sydney \\ Australia \\ Email: Robin.Braun@uts.edu.au
}

\begin{abstract}
The concept of 5G is regarded to gain 1000x data speed with 20 Billion devices (IoT) connected to the network which will literary connect everything. From the network point of view, lower latency combined with high flexibility is not just limited to the concept of $5 \mathrm{G}$ but already on the way to be implemented in real network by 2020 . In a cellular network, Device to Device (D2D) communication has been viewed as a promising technology to overcome many existed problems especially the capacity and quality considering the scarce spectrum resource. However, this comes at the price of huge interference and complex mobility issues although it was initially proposed as a new paradigm to enhance the network performance. Cellular devices without smooth mobility readiness are hardly acceptable. A smart mobility system in cellular inband underlay D2D communication should have lower latency, lower power consumption, and higher data rates. My proposition is to review the existing mobility management system for the LTE-Advanced technology and propose a unique algorithm over the existing proposal so that lower signalling overhead and lower delay along with uninterrupted D2D communication are guaranteed for the 5G cellular communication system by 2020 .
\end{abstract}

\section{INTRODUCTION}

The telecommunication system is still the key to success and survival from the very pre-historic man with fire signal to the current smart-phone with the millions of applications. Fifth Generation (5G) mobile network refers to the next major phase of mobile telecommunications system beyond the currently available IMT-Advanced/4G standards. 5G is expected to fulfil major demands of data services in telecommunication system along with new era of Internet of Things (IoT). A lot of researchers have proposed the probable technological enhancement for the $5 \mathrm{G}$ network. Some big projects like METIS [1] and 5GNOW [2] already progressed significantly towards 5G. In [3], authors highlight five disruptive technology directions for $5 \mathrm{G}$ network which are device-centric architecture, milimeter wave (mmWave), massive MIMO, smarter devices, native support for machine to machine (M2M) communications. A multi-tier 5G wireless cellular network from an interference management perspective is suggested in [4]. In this paper, visions and requirements for the 5G multi-tier network are discussed that are data rate and latency, Machine Type Communication (MTC) devices,
mmWave communication, multiple RATS, base station densification, prioritized spectrum access, network-assisted Device to Device (D2D) communication, energy harvesting for energy-efficient communication. 3D beamforming [5] is another scope of 5G. 5G network is assumed to change into device-centric architecture [3]. Uniform network solution is the trend and challenge for the 5G network. Wireless communications without mobility could hardly be imagined. Mobility management for the any wireless communications has been regarded as the high priority issue. We have almost smooth mobility management for the latest telecommunications system like LTE-A. However, mobility for the D2D devices is not so easy to compare with the currently available techniques. The aim of the paper is to establish an algorithm that will facilitate the smooth mobility system for the D2D devices.An excellent survey [6] explains almost all the basics of D2D communication in a cellular network. It also highlights the major areas in this regard. Survey papers [7] [8] also describe the overall concepts and research ideas in D2D communication.

The rest of the paper is structured such as next section discusses mobility issues of D2D communications. A noble approach of mobility issue of D2D communication is presented in section three. Finally, section four concludes the paper with focusing the major works done.

\section{Mobility issues of D2D COMmunications}

D2D communication takes place when two devices communicate directly without taking major help from the base station. For any wireless communication system, mobility management is a big issue. When two devices are active, any or both devices might change its location, and when they are in the cellular mode, it is not difficult to keep the service uninterrupted. However, when it is in D2D mode, mobility management becomes crucial since a proper algorithm is yet to develop that can handle it without interruption. A low latency reliable data communication between two devices while moving is a challenging task that is yet to be developed. Very few papers discussed on this so far. When several base 
stations are involved to make the handover, the latency could be increased for exchanging the controlling signal that makes D2D communications infeasible while moving. A low latency, smooth mobility management is very crucial because, beside the human-centric, one of the very important applications of this communication is vehicle-to-vehicle (V2V) communication and a bit longer latency might cause severe damage in this regard. Paper [9] propose some mobility management solutions that shows expected gains under certain assumptions for small cell network. Authors categorized two smart solutions that could reduce the negative impact.

\section{A. D2D-Aware Handover Solution}

This solution is proposed to reduce the latency by minimizing signalling overhead. In normal communication when two devices are stable in a place, a lot of overhead is involved while UE1 is moving from one location to another location that is served by another base station. This is not feasible in D2D communications because of the higher latency. Authors propose a group handover algorithm so that latency is reduced greatly. A3 event has been considered where $R S R P_{\text {target }}-R S R P_{\text {source }}>$ offset. They propose that the control of both devices should be under the same base station and until and unless this condition is satisfied they propose not to perform the handover for the individual device. Paper [9] shows the detail steps to perform this task.

\section{B. D2D-Triggered Handover Solution}

Authors propose a signalling flow chart in [9] of D2Dtriggered handover that shows how a target cell handover the D2D control to a new D2D user to join the group communication. In this type of solution, authors intend to cluster the total members of a D2D group within a minimum number of BSs or cells so that network signalling overheads caused by the interBS communication, e.g., D2D radio resource information are reduced.

\section{Proposed Model of Mobility Management}

In this part, we consider the TDD configurations that is already used for LTE-A. So, standard power control mechanism for the TDD will be applicable accordingly. For the convenient, we assume that DUEs use the uplink resources of a mobile cellular network. The purpose of our proposal is to increase the sum-rate that should remain same while moving.

Consider an area where different users are located within a cell by a single basestation. Fig. 1 shows a model where different links and channels are shown where the functions of the small cells are ignored. We also consider that the cellular and D2D users are in active mode simultaneously. That means while cellular users communicate with eNodeB using uplink frame, D2D users also communicate with each other at the same time with same uplink resources so that spectrum utilization is confirmed maximum. In this case, when device 2,3 and 4 are in active, eNodeB is silent and when eNodeB transmits, device 2, 3 and 4 are silent. We

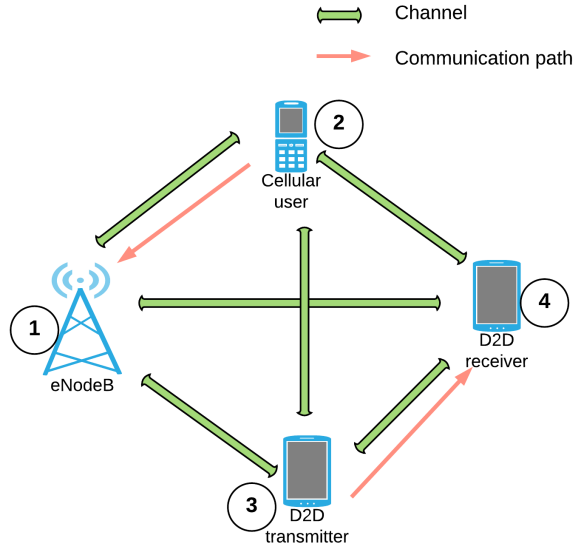

Fig. 1. D2D communications and possible channel links within a cell.

assume one eNB and three devices and they are numbered $p=1,2,3,4$ and $q=1,2,3,4$ respectively as per Fig. 1 . The link is indicated $X_{p q}$ where $p$ is the position of first device and $q$ is the position of second device of a link.

Regarding the channel links, some channels are effective and some might be ignored as they are negligible compared with others. Generally following links could be ignored irrespective of the conditions, as common for both CUEs and DUEs, for CUEs only, for DUEs only.

$$
\begin{array}{r}
X_{p q}=0 \text { if } p=1,4 \text { and } q=4,1 \text { respectively } \\
\text { or } p=2,3 \text { and } q=3,2 \text { respectively }
\end{array}
$$

For the both cellular and D2D modes,

$$
\begin{array}{r}
X_{p q}=0 \text { if } p=1,3 \text { and } q=3,1 \text { respectively } \\
\text { or } p=2,4 \text { and } q=4,2 \text { respectively }
\end{array}
$$

Sum rate for the cellular

$$
r_{\text {Cellular }}=B \log _{2}\left(1+\frac{S_{\text {Cellular }}}{\sum_{p} \sum_{q} X_{p q}+N_{0}}\right)
$$

Sum rate for the DUEs

$$
r_{\mathrm{D} 2 \mathrm{D}}=B \log _{2}\left(1+\frac{S_{\mathrm{D} 2 \mathrm{D}}}{\sum_{p} \sum_{q} X_{p q}+N_{0}}\right)
$$

Total sum-rate can be calculated as

$$
R_{\text {Sum }}=r_{\text {Cellular }}+r_{\mathrm{D} 2 \mathrm{D}}
$$

where, B is the bandwidth of each subchannel. $S_{\mathrm{D} 2 \mathrm{D}}$ is the signal gain of D2D users and $S_{\text {Cellular }}$ is the signal gain of the cellular user. $N_{0}$ is the additive noise. Our proposal considers the paper [10] where an operational procedure of an LTE network that supports D2D communications is proposed. Fig. 2 shows the brief line diagram how this proposal works. Our algorithm extends this procedure which should support the mobility of DUEs without compromising the quality. Consider a total number of CUEs and DUEs pair (each pair 
consists of two D2D users, one is the transmitter, and another is the receiver) are $N_{c}$ and $N_{d}$ respectively. To improve the spectrum efficiency, we consider the same uplink resources for both CUEs and DUEs.

We propose an extended algorithm of this model that enables the mobility functionality of D2D users in a $5 \mathrm{G}$ cellular network. Fig. 3 shows the propose model where mobility direction, relevant channel gain, and communication paths have been indicated. Some links have been ignored as they are negligible according to the previous equations. Picture also shows the notation of channel gains. Based on the moving opportunities, we consider four scenarios as follows:

- $D U E T_{X}$ moving towards another eNB. Here, $D U E T_{X}$ is the transmitter of the D2D pair.

- $D U E R_{X}$ moving towards another eNB. Here, $D U E R_{X}$ is the receiver of the D2D pair.

- Both $D U E T_{X}$ and $D U E R_{X}$ are moving into same eNB.

- Both $D U E T_{X}$ and $D U E R_{X}$ are moving apart from each other and exiting the current eNB serving area.

For the convenient, we consider only first condition in this paper where D2D transmitter is moving towards another eNB as in Fig. 3. Rest of the conditions are to explore in future.

eNodeB always collects the channel state/gain information and allocates the resources along with transmit power of CUEs and DUEs. Assume that total $N_{c} \times N_{d}$ resource sharing pair for the current cell and target cell. One resource sharing pair consists of one D2D pair and one CUE within the same cell. Consider $f_{i j}$ where $i \in 1, . . N_{c}$ and $j \in 1, . . N_{d}$ is a specific resource sharing pair composed of $i$ th CUE and $j$ th DUE pair. For the convenient to indicate gain of a link, $d_{j}^{R}$ is referred to as $\mathrm{D} 2 \mathrm{D}$ receiver, $d_{j}^{T}$ is the $\mathrm{D} 2 \mathrm{D}$ transmitter, $c_{i}^{T}$ is the cellular user of target cell, $c_{i}^{S}$ is the cellular user of source cell, $e_{o}^{T}$ is the target cell, $e_{o}^{S}$ is the source cell. Also, the cellular user is indicated as $C U E_{i}^{S}$ for the source cell and $C U E_{i}^{T}$ for target cell. $D U E T_{x j}$ is the D2D transmitter and $D U E R_{x j}$ is the receiver of the same cell. The new gain between the D2D users after moving is $\bar{G} d_{j}^{T} d_{j}^{R}$.

Assuming the same amount of source sharing pair exist after the moving takes place in target cell, i.e., DUEs moving into the new cell would not change the number of total CUEs and DUEs. So the values of $i$ and $j$ will remain the same number before the handover in the source cell and after the handover to the target eNodeB. Fig. 4 shows the steps of the handover management during the D2D communications. Handover process is initiated at $(n+2)$ th time whereas previous time is for the usual D2D communications without any mobility scope according to the paper [10].

Once the target eNodeB starts the handover preparation, both D2D users calculates the channel gains $A=G c_{i}^{T} d_{j}^{R}$ for all $i$ and $B=\bar{G} d_{j}^{T} d_{j}^{R}$ for all $j$ in the target cell. Parallelly, cellular user at the target cell calculates the gain $C=G c_{i}^{T} e_{o}^{T}$

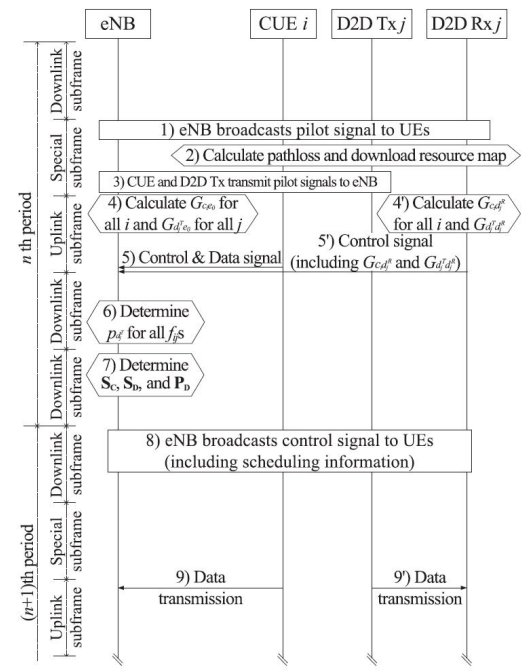

Fig. 2. A D2D operation model proposed in [10]

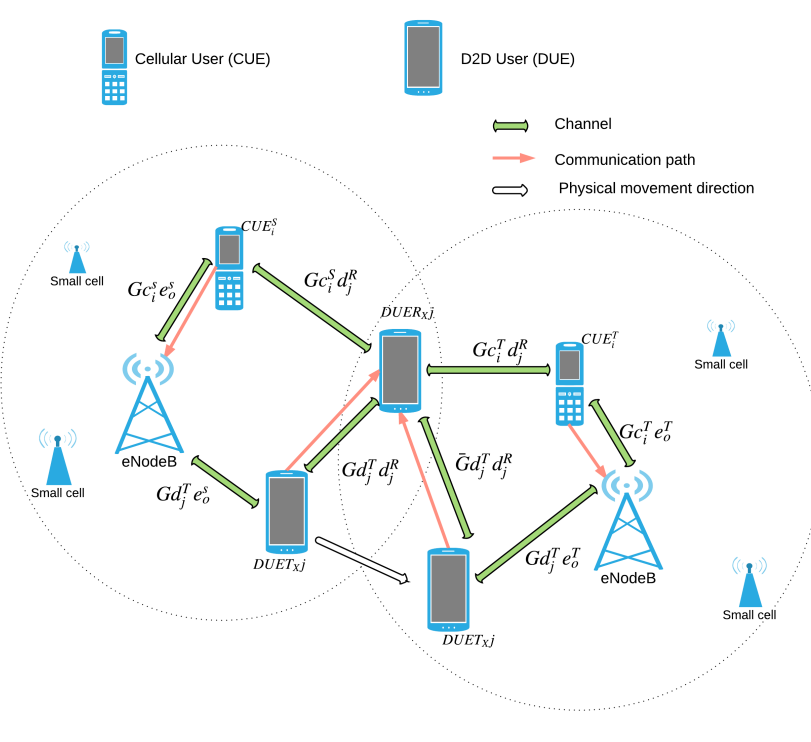

Fig. 3. Proposed mobility model.

for all $i$ and target eNodeB calculates $D=G d_{j}^{T} e_{o}^{T}$ for all $j$. During the data transmission, periodic measurement broadcast signals are sent to all CUEs and DUEs. In this measurement signals, path loss and relevant parameters data are sent and based on these information D2D users or eNode takes the decision to make the handover. The relevant channel gains are indicated in Fig. 3 where channel gain between $C U E_{i}^{S}$ and $D U E T_{x j}$, and eNB and $D U E R_{x j}$ are neglected as indicated earlier in equations. This is to be noted that when one of the D2D users is moved to a target cell, the procedures for the resource allocations and scheduling are the same as it already did with the source cells. Target eNB determines the transmit power $\bar{P} d_{j}^{T}$ for the D2D users. Based on the power allocation, eNB calculate the matrix $\overline{\mathbf{S}}_{C}$ and $\overline{\mathbf{S}}_{D}$ for assigning 


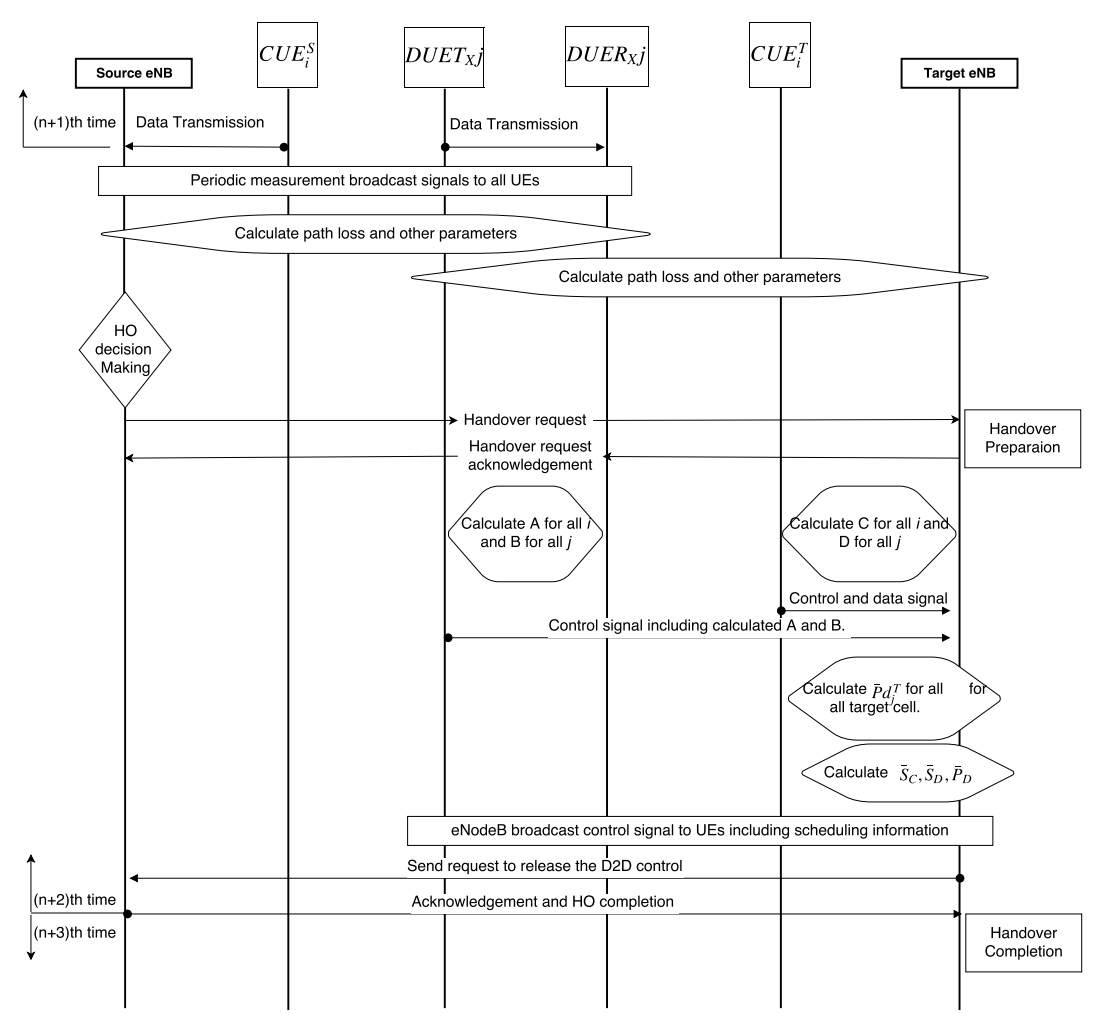

Fig. 4. Line diagram of proposed model

the subchannel to the cellular users and D2D users. Finally, eNB constructs the matrix $\overline{\mathbf{P}}_{D}=\overline{\mathbf{S}}_{C} \overline{\mathbf{P}}_{F}$ for the all users within the cell, where, $\overline{\mathbf{P}}_{F}$ is the matrix consists of the values $\bar{p} d_{j}^{T}$ for each $f_{i j}$. Accordingly to the paper [10], final transmit power for D2D users on each subchannel is calculated by the equation

$$
\mathbf{P}_{D}=\mathbf{S}_{D} \circ \overline{\mathbf{P}}_{\mathbf{D}}
$$

where, $\mathbf{S}_{D}$ is the result of resource scheduling for DUEs at the target cell and $\circ$ is a Hadamard operator.

Since all the calculations related with the target cells are almost similar to the source cell during the normal operation of D2D mode, the only latency required is for taking the handover decision and relevant gain calculations which is as like as usual handover operation. The total sum-rate would be maximum and as both the cellular user and D2D users use same uplink resources, the spectrum efficiency would be the highest.

\section{CONCLUSION}

In this paper, we tried to review the entire D2D system along with the technologies that might fit with the 5G system. Starting from the prospective different technologies for the 5G cellular systems, we tried to highlight the facts of D2D communications and explored the recent papers for the mobility management of same system. Finally we proposed a model for the D2D communication that might be a future of entire mobility system of D2D communication in 5G. As similar proposal has not been investigated earlier, we call it a novel approach. Details simulation results along with some pros and cons are to explore in our next paper.

\section{REFERENCES}

[1] "FP7 European Project 317669 METIS (Mobile and WIreless Communications Enablers for the Twenty-Twenty Information Society)." www.metis2020.com. [Online; accessed 19-January-2016].

[2] "FP7 European Project 318555 5G NOW (5th Generation NonOrthogonal Waveforms for Asynchronous Signalling)." www.5gnow.eu. [Online; accessed 19-January-2016].

[3] F. Boccardi, R. W. Heath, A. Lozano, T. L. Marzetta, and P. Popovski, "Five disruptive technology directions for 5g," Communications Magazine, IEEE, vol. 52, no. 2, pp. 74-80, 2014.

[4] E. Hossain, M. Rasti, H. Tabassum, and A. Abdelnasser, "Evolution toward 5g multi-tier cellular wireless networks: An interference management perspective," Wireless Communications, IEEE, vol. 21, no. 3, pp. $118-127,2014$.

[5] S. Barua, S. C. Lam, P. Ghosa, S. Xing, and K. Sandrasegaran, "A survey of direction of arrival estimation techniques and implementation of channel estimation based on scme," in ECTI-CON, 2015, pp. 1-5.

[6] A. Asadi, Q. Wang, and V. Mancuso, "A survey on device-to-device communication in cellular networks," Communications Surveys \& Tutorials, IEEE, vol. 16, no. 4, pp. 1801-1819, 2014.

[7] K. Doppler, M. Rinne, C. Wijting, C. B. Ribeiro, and K. Hugl, "Deviceto-device communication as an underlay to lte-advanced networks," Communications Magazine, IEEE, vol. 47, no. 12, pp. 42-49, 2009.

[8] L. Wei, R. Hu, Y. Qian, and G. Wu, "Enable device-to-device communications underlaying cellular networks: challenges and research aspects," Communications Magazine, IEEE, vol. 52, no. 6, pp. 90-96, 2014.

[9] O. N. Yilmaz, Z. Li, K. Valkealahti, M. A. Uusitalo, M. Moisio, P. Lundén, and C. Wijting, "Smart mobility management for d2d communications in 5g networks," in WCNCW, 2014 IEEE, pp. 219-223.

[10] G. Jaheon, S. J. Bae, S. F. Hasan, and M. Y. Chung, "A combined power control and resource allocation scheme for $\mathrm{d} 2 \mathrm{~d}$ communication underlaying an lte-advanced system," IEICE Transactions on Communications, vol. 96, no. 10, pp. 2683-2692, 2013. 